\title{
ON WEIGHTED INEQUALITIES FOR CERTAIN FRACTIONAL INTEGRAL OPERATORS
}

\author{
R. K. RAINA
}

Received 31 December 2005; Revised 8 May 2006; Accepted 9 May 2006

This paper considers the modified fractional integral operators involving the Gauss hypergeometric function and obtains weighted inequalities for these operators. Multidimensional fractional integral operators involving the $\mathrm{H}$-function are also introduced.

Copyright (c) 2006 Hindawi Publishing Corporation. All rights reserved.

\section{Introduction and preliminaries}

Tuan and Saigo [7] introduced the multidimensional modified fractional integrals of or$\operatorname{der} \alpha(\operatorname{Re}(\alpha)>0)$ by

$$
\begin{aligned}
& X_{+; n}^{\alpha} f(x)=\frac{1}{\Gamma(\alpha+1)} \mathbf{D}^{\mathbf{n}} \int_{\mathbb{R}_{+}^{\mathrm{n}}}\left[\min \left\{\frac{x_{1}}{t_{1}}, \ldots, \frac{x_{n}}{t_{n}}\right\}-1\right]_{+}^{\alpha} f(t) d t, \\
& X_{-; n}^{\alpha} f(x)=\frac{(-1)^{n}}{\Gamma(\alpha+1)} \mathbf{D}^{\mathbf{n}} \int_{\mathbb{R}_{+}^{\mathrm{n}}}\left[1-\max \left\{\frac{x_{1}}{t_{1}}, \ldots, \frac{x_{n}}{t_{n}}\right\}\right]_{+}^{\alpha} f(t) d t,
\end{aligned}
$$

where $\mathbb{R}_{+}^{\mathbf{n}}=\left\{\left(t_{1}, \ldots, t_{n}\right) \mid t_{i}>0(i=1, \ldots, n)\right\}, \varphi_{+}(x)$ is a real-valued function defined in terms of the function $\varphi(x)$ by

$$
\varphi_{+}(x)= \begin{cases}\varphi(x), & \varphi(x)>0, \\ 0, & \varphi(x) \leq 0,\end{cases}
$$

and $\mathbf{D}^{\mathbf{n}}$ denotes the derivative operator $\partial^{n} / \partial x_{1}, \ldots, \partial x_{n}$.

The operators in (1.1) provide multidimensional generalizations to the well-known one-dimensional Riemann-Liouville and Weyl fractional integral operators defined in [5] (see also [1]). The paper [7] considers several formulas and interesting properties of (1.1). By invoking the Gauss hypergeometric function ${ }_{2} F_{1}(\alpha, \beta ; \gamma ; x)$, the following generalizations of the multidimensional modified integral operators $(1.1)$ of order $\alpha(\operatorname{Re}(\alpha)>0)$ 
were studied in [6]:

$$
\begin{aligned}
S_{+; n}^{\alpha, \beta, \gamma} f(x)= & \frac{1}{\Gamma(\alpha+1)} \mathbf{D}^{\mathbf{n}} \int_{\mathbb{R}_{+}^{\mathrm{n}}}\left[\min \left\{\frac{x_{1}}{t_{1}}, \ldots, \frac{x_{n}}{t_{n}}\right\}-1\right]_{+}^{\alpha} \\
& \cdot{ }_{2} F_{1}\left(\alpha+\beta, \alpha+\eta ; 1+\alpha ; 1-\min \left\{\frac{x_{1}}{t_{1}}, \ldots, \frac{x_{n}}{t_{n}}\right\}\right) f(t) d t, \\
S_{-; n}^{\alpha, \beta, \gamma} f(x)= & \frac{(-1)^{n}}{\Gamma(\alpha+1)} \mathbf{D}^{\mathbf{n}} \int_{\mathbb{R}_{+}^{\mathrm{n}}}\left[1-\max \left\{\frac{x_{1}}{t_{1}}, \ldots, \frac{x_{n}}{t_{n}}\right\}\right]_{+}^{\alpha} \\
& \cdot{ }_{2} F_{1}\left(\alpha+\beta,-\eta ; 1+\alpha ; 1-\max \left\{\frac{x_{1}}{t_{1}}, \ldots, \frac{x_{n}}{t_{n}}\right\}\right) f(t) d t .
\end{aligned}
$$

For $\beta=-\alpha$, the operators (1.3) and (1.4) reduce to the modified integral operators defined in (1.1), respectively. In [8], the integral operators $X_{+; n}^{\alpha} f(x)$ and $X_{-; n}^{\alpha} f(x)$ defined on the space $M_{\gamma}\left(\mathbb{R}_{+}^{\mathbf{n}}\right)$ are shown to satisfy some $L_{p}-L_{q}$ weighted inequalities. The space $M_{\gamma}\left(\mathbb{R}_{+}^{\mathbf{n}}\right)$ represents the space of functions $f$ which are defined on $\mathbb{R}_{+}^{\mathbf{n}}$, and are entire functions of exponential type (see [7]). The present paper is devoted to finding inequalities for the generalized multidimensional modified integral operators (1.3) and (1.4) by making use of the inequality stated in [8] (which was established with the aid of Pitt's inequality). Multidimensional operators have also been studied in $[3,4]$.

\section{Inequalities for operators (1.3) and (1.4)}

If $(\mathscr{K} f)(x)$ denotes the integral operator

$$
(\mathscr{K} f)(x)=\int_{\mathbb{R}_{+}^{\mathrm{n}}} k(x y) f(y) d y
$$

then following [8], we have

$$
\int_{\mathbb{R}_{+}^{\mathrm{n}}} k(x y) f(y) d y=\frac{1}{(2 \pi i)^{n}} \int_{(1 / 2)} k^{*}(s) f^{*}(1-s) x^{-s} d s
$$

where the integral over (1/2) stands for the multiple integral

$$
\int_{(1 / 2)}=\int_{1 / 2-i \infty}^{1 / 2+i \infty} \cdots \int_{1 / 2-i \infty}^{1 / 2+i \infty}
$$

and $k^{*}(s)$ and $f^{*}(1-s)$ are the Mellin transforms of the functions $k(y)$ and $f(y)$, respectively. It is proved in [8] that if

$$
\left|k^{*}(s)\right| \leq C|s|^{-\alpha}\left(s \in\left(\frac{1}{2}\right), \alpha \geq 0\right)
$$

then there holds the inequality

$$
\left\|\left.|\log y-t|^{-b} y^{1 / 2-1 / r}(\mathscr{K} f)(y)\right|_{L_{r}\left(\mathbb{R}_{+}^{\mathrm{n}}\right)} \leq C|||\log y-t|^{d} y^{1 / 2-1 / q} f(y)\right\|_{L_{q}\left(\mathbb{R}_{+}^{\mathrm{n}}\right)},
$$


or equivalently,

$$
\left\||\log y-t|^{-b} y^{1 / 2-1 / r}(\mathscr{K} f)(y)\right\|_{L_{r}\left(\mathbb{R}_{+}^{\mathrm{n}}\right)} \leq C|||\log y-t|^{a-b+n(1 / r-1 / q)} y^{1 / 2-1 / q} f(y) \|_{L_{q}\left(\mathbb{R}_{+}^{\mathrm{n}}\right)},
$$

for all $t \in \mathbb{R}_{+}^{\mathbf{n}}$, provided that

$$
\left[\begin{array}{c}
\max \left\{\frac{2}{r}, \frac{\alpha}{n}+\frac{1}{r}\right\}-1 \leq \frac{b}{n} \leq \min \left\{0, \frac{\alpha}{n}-\frac{1}{q}, \frac{\alpha}{n}+\frac{1}{q}-1\right\}+\frac{1}{r}, \\
\max \left\{0, n\left(\frac{1}{r}+\frac{1}{q}-1\right)\right\} \leq \alpha \leq n, 1<q \leq r<\infty .
\end{array}\right] .
$$

In the paper [6], it was established that if

$$
\operatorname{Re}(\alpha)>0, \quad \operatorname{Re}\left(h_{j}\right)<\frac{1}{2} \quad(j=1, \ldots, n), \quad \sum_{j=1}^{n} \operatorname{Re}\left(h_{j}\right)<\frac{n}{2}+\min \{\operatorname{Re}(\beta), \operatorname{Re}(\eta)\},
$$

then the operator $x^{h} S_{+; n}^{\alpha, \beta, \gamma} x^{-h} f(x)$ is a homeomorphism of the space $M_{1 / 2}\left(\mathbb{R}_{+}^{\mathbf{n}}\right)$ onto itself, and

$$
\begin{aligned}
& x^{h} S_{+; n}^{\alpha, \beta, \gamma} x^{-h} f(x) \\
& \quad=\frac{1}{(2 \pi i)^{n}} \int_{(1 / 2)} \frac{\Gamma\left(\beta+n-\sum_{j=1}^{n} h_{j}-\sum_{j=1}^{n} s_{j}\right) \Gamma\left(\eta+n-\sum_{j=1}^{n} h_{j}-\sum_{j=1}^{n} s_{j}\right)}{\Gamma\left(n-\sum_{j=1}^{n} h_{j}-\sum_{j=1}^{n} s_{j}\right) \Gamma\left(\alpha+\beta+\eta+n-\sum_{j=1}^{n} h_{j}-\sum_{j=1}^{n} s_{j}\right)} f^{*}(s) x^{-s} d s .
\end{aligned}
$$

We note that

$$
\frac{\Gamma\left(\beta+n-\sum_{j=1}^{n} h_{j}-\sum_{j=1}^{n} s_{j}\right) \Gamma\left(\eta+n-\sum_{j=1}^{n} h_{j}-\sum_{j=1}^{n} s_{j}\right)}{\Gamma\left(n-\sum_{j=1}^{n} h_{j}-\sum_{j=1}^{n} s_{j}\right) \Gamma\left(\alpha+\beta+\eta+n-\sum_{j=1}^{n} h_{j}-\sum_{j=1}^{n} s_{j}\right)}=O\left(|s|^{-\alpha}\right),
$$

so we can apply the inequality (2.6) to the multidimensional operator defined by (2.9), which leads to

$$
\begin{aligned}
& \quad\left\||\log y-t|^{-b} y^{1 / 2-1 / r+h} S_{+; n}^{\alpha, \beta, \gamma} y^{-h} f(y)\right\|_{L_{r}\left(\mathbb{R}_{+}^{\mathrm{n}}\right)} \\
& \quad \leq C|||\log y-t|^{a-b+n(1 / r-1 / q)} y^{1 / 2-1 / q} f(y) \|_{L_{q}\left(\mathbb{R}_{+}^{\mathrm{n}}\right)},
\end{aligned}
$$

valid for all $t \in \mathbb{R}_{+}^{\mathbf{n}}$, provided that the constraints (2.7) and (2.8) are satisfied. On the other hand, (see [6]) if

$$
\operatorname{Re}(\alpha)>0, \quad \operatorname{Re}\left(h_{j}\right)>\frac{1}{2} \quad(j=1, \ldots, n), \quad \sum_{j=1}^{n} \operatorname{Re}\left(h_{j}\right)>\frac{n}{2}+\operatorname{Re}(\beta-\eta)-1,
$$


then the operator $x^{h} S_{-; n}^{\alpha, \beta, \gamma} x^{-h} f(x)$ is a homeomorphism of the space $M_{1 / 2}\left(\mathbb{R}_{+}^{\mathbf{n}}\right)$ onto itself, and we obtain

$$
\begin{aligned}
& x^{h} S_{-; n}^{\alpha, \beta, \gamma} x^{-h} f(x) \\
& =\frac{1}{(2 \pi i)^{n}} \int_{(1 / 2)} \frac{\Gamma\left(1-n+\sum_{j=1}^{n} h_{j}+\sum_{j=1}^{n} s_{j}\right) \Gamma\left(1-\beta+\eta-n+\sum_{j=1}^{n} h_{j}+\sum_{j=1}^{n} s_{j}\right)}{\Gamma\left(1-\beta-n+\sum_{j=1}^{n} h_{j}+\sum_{j=1}^{n} s_{j}\right) \Gamma\left(1+\alpha+\eta-n+\sum_{j=1}^{n} h_{j}+\sum_{j=1}^{n} s_{j}\right)} \\
& \quad \times f^{*}(s) x^{-s} d s .
\end{aligned}
$$

By noting the estimate that

$$
\frac{\Gamma\left(1-n+\sum_{j=1}^{n} h_{j}+\sum_{j=1}^{n} s_{j}\right) \Gamma\left(1-\beta+\eta-n+\sum_{j=1}^{n} h_{j}+\sum_{j=1}^{n} s_{j}\right)}{\Gamma\left(1-\beta-n+\sum_{j=1}^{n} h_{j}+\sum_{j=1}^{n} s_{j}\right) \Gamma\left(1+\alpha+\eta-n+\sum_{j=1}^{n} h_{j}+\sum_{j=1}^{n} s_{j}\right)}=O\left(|s|^{-\alpha}\right),
$$

we again apply the inequality (2.6) to the multidimensional operator defined by (2.13) to get

$$
\begin{aligned}
& \left\||\log y-t|^{-b} y^{1 / 2-1 / r+h} S_{-; n}^{\alpha, \beta, \gamma} y^{-h} f(y)\right\|_{L_{r}\left(\mathbb{R}_{+}^{\mathrm{n}}\right)} \\
& \quad \leq C|| t|\log y-t|^{a-b+n(1 / r-1 / q)} y^{1 / 2-1 / q} f(y) \|_{L_{q}\left(\mathbb{R}_{+}^{\mathrm{n}}\right)},
\end{aligned}
$$

valid for all $t \in \mathbb{R}_{+}^{\mathbf{n}}$, provided that the constraints (2.7) and (2.12) are satisfied.

\section{Classes of multidimensional operators}

We introduce the following classes of multidimensional modified fractional integral operators involving the well-known H-function [2, Section 8.3] (see also [1, page 343]) defined by

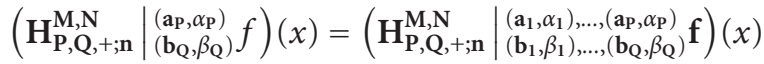

$$
\begin{aligned}
& =\mathbf{D}^{\mathbf{n}} \int_{\mathbb{R}_{+}^{\mathbf{n}}} H_{P, Q}^{M, N}\left[\min \left\{\frac{x_{1}}{t_{1}}, \ldots, \frac{x_{n}}{t_{n}}\right\} \mid \begin{array}{c}
\left(a_{P}, \alpha_{P}\right) \\
\left(b_{Q}, \beta_{Q}\right)
\end{array}\right] f(t) d t,
\end{aligned}
$$

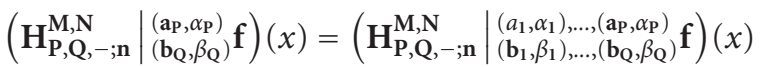

$$
\begin{aligned}
& =(-1)^{n} \mathbf{D}^{\mathbf{n}} \int_{\mathbb{R}_{+}^{n}} H_{P, Q}^{M, N}\left[\max \left\{\frac{x_{1}}{t_{1}}, \ldots, \frac{x_{n}}{t_{n}}\right\} \mid \begin{array}{c}
\left(a_{P}, \alpha_{P}\right) \\
\left(b_{Q}, \beta_{Q}\right)
\end{array}\right] f(t) d t,
\end{aligned}
$$

where we assume that the parameters of the $\mathrm{H}$-function involved in (3.1) and (3.2) satisfy the existence conditions as given in [2].

The special cases of the operators of interest in this paper are the operators which emerge from (3.1) and (3.2) in the case when $N=0, P=M, Q=M$, and the parameters $\alpha_{1}=\alpha_{2}=\cdots=\alpha_{m}=1$, and $\beta_{1}=\beta_{2}=\cdots=\beta_{m}=1$. Thus, we have the following multidimensional fractional integral operators (defined in terms of Meijer's G-function) 
(see [6]):

$$
\begin{aligned}
\left(\mathbf{G}_{+; \mathbf{n}}^{\left(\mathbf{a}_{\mathbf{m}}\right) ;\left(\mathbf{b}_{\mathbf{m}}\right)} \mathbf{f}\right)(x) & =\left(\mathbf{G}_{+; \mathbf{n}}^{\left(\mathbf{a}_{1}, \ldots, \mathbf{a}_{\mathbf{m}}\right) ;\left(\mathbf{b}_{1}, \ldots, \mathbf{b}_{\mathbf{m}}\right)} \mathbf{f}\right)(x) \\
& =\mathbf{D}^{\mathbf{n}} \int_{\mathbb{R}_{+}^{\mathbf{n}}} G_{m, m}^{m, 0}\left[\left.\min \left\{\frac{x_{1}}{t_{1}}, \ldots, \frac{x_{n}}{t_{n}}\right\}\right|_{\left(b_{m}\right)} ^{\left(a_{m}\right)}\right] f(t) d t, \\
\left(\mathbf{G}_{-; \mathbf{n}}^{\left(\mathbf{a}_{\mathbf{m}}\right) ;\left(\mathbf{b}_{\mathbf{m}}\right)} \mathbf{f}\right)(x) & =\left(\mathbf{G}_{+; \mathbf{n}}^{\left(\mathbf{a}_{1}, \ldots, \mathbf{a}_{\mathbf{m}}\right) ;\left(\mathbf{b}_{1}, \ldots, \mathbf{b}_{\mathbf{m}}\right)} \mathbf{f}\right)(x) \\
& =(-1)^{n} \mathbf{D}^{\mathbf{n}} \int_{\mathbb{R}_{+}^{\mathbf{n}}} G_{m, m}^{m, 0}\left[\left.\max \left\{\frac{x_{1}}{t_{1}}, \ldots, \frac{x_{n}}{t_{n}}\right\}\right|_{\left(b_{m}\right)} ^{\left(a_{m}\right)}\right] f(t) d t .
\end{aligned}
$$

By setting the parameters

$$
m=2, \quad a_{1}=1-\beta, \quad a_{2}=1-\eta, \quad b_{1}=1-\alpha-\beta-\eta, \quad b_{2}=0,
$$

in (3.1), and

$$
m=2, \quad a_{1}=1-\beta, \quad a_{2}=1+\alpha+\eta, \quad b_{1}=1-\beta+\eta, \quad b_{2}=0,
$$

in (3.2), and noting the relation (see [1, equation (1.1.18), page 18] )

$$
\begin{aligned}
& G_{2,2}^{2,0}\left[\sigma \mid \begin{array}{c}
a_{1}, a_{2} \\
b_{1}, b_{2}
\end{array}\right]=\frac{\sigma^{b_{2}}(1-\sigma)^{a_{1}+a_{2}-b_{1}-b_{2}-1}}{\Gamma\left(a_{1}+a_{2}-b_{1}-b_{2}\right)} \\
& { }_{2} F_{1}\left(a_{2}-b_{1}, a_{1}-b_{1} ; a_{1}+a_{2}-b_{1}-b_{2} ; 1-\sigma\right) \quad(\sigma<1) \text {, }
\end{aligned}
$$

we observe the following relationships:

$$
\begin{gathered}
\left(\mathbf{G}_{+; \mathbf{n}}^{(\mathbf{1}-\beta, \mathbf{1}-\eta) ;(\mathbf{1}-\alpha-\beta-\eta, \mathbf{0})} \mathbf{f}\right)(x)=(-1)^{\alpha} S_{+; n}^{\alpha, \beta, \gamma} f(x), \\
\left(\mathbf{G}_{-; \mathbf{n}}^{(\mathbf{1}-\beta, \mathbf{1}+\alpha+\eta) ;(\mathbf{1}-\beta+\eta, \mathbf{0})} \mathbf{f}\right)(x)=S_{-; n}^{\alpha, \beta, \gamma} f(x),
\end{gathered}
$$

in terms of the multidimensional modified fractional integral operators (1.3) and (1.4).

We state below two useful lemmas concerning the multidimensional Mellin transform of the functions $f\left(\max \left[x_{1}, \ldots, x_{n}\right]\right)$ and $f\left(\min \left[x_{1}, \ldots, x_{n}\right]\right)$ (see $\left.[3,6]\right)$.

Lemma 3.1. Let $\operatorname{Re}\left(s_{j}\right)>0(j=1, \ldots, n)$ and let $\tau^{s \cdot 1-1} f(\tau) \in L_{1}\left(\mathbb{R}_{+}\right)$, then

$$
\int_{\mathbb{R}_{+}^{n}} x^{s-1} f\left(\max \left[x_{1}, \ldots, x_{n}\right]\right) d x=\frac{|s|}{s^{1}} f^{*}(|s|),
$$

where $s^{1}$ denotes the product $s_{1}, \ldots, s_{n}$, and $|s|=s_{1}+\cdots+s_{n}$.

Lemma 3.2. Let $\operatorname{Re}\left(s_{j}\right)<0(j=1, \ldots, n)$ and let $\tau^{s \cdot 1-1} f(\tau) \in L_{1}\left(\mathbb{R}_{+}\right)$, then

$$
\int_{\mathbb{R}_{+}^{\mathrm{n}}} x^{s-1} f\left(\min \left[x_{1}, \ldots, x_{n}\right]\right) d x=(-1)^{n-1} \frac{|s|}{s^{1}} f^{*}(|s|) .
$$


6 Inequalities for certain integral operators

Making use of (3.1), we have

$$
\begin{aligned}
\left(\mathbf{H}_{\mathbf{P}, \mathbf{Q},+; \mathbf{n}}^{\mathrm{M}, \mathbf{N}} \mid \underset{\left(\mathbf{b}_{\mathbf{Q}}, \beta_{\mathbf{Q}}\right)}{\left(\mathbf{a}_{\mathbf{p}}, \alpha_{\mathbf{P}}\right)} \mathbf{x}^{-\mathbf{s}}\right)(x) & =\mathbf{D}^{\mathbf{n}} \int_{\mathbb{R}_{+}^{\mathbf{n}}} H_{P, Q}^{M, N}\left[\left.\min \left\{\frac{x_{1}}{t_{1}}, \ldots, \frac{x_{n}}{t_{n}}\right\}\right|_{\left(b_{Q}, \beta_{\mathbf{Q}}\right)} ^{\left(a_{P}, \alpha_{P}\right)}\right] t^{-s} d t \\
& =\mathbf{D}^{\mathbf{n}} x^{1-s} \int_{\mathbb{R}_{+}^{\mathbf{n}}} t^{s-2} H_{P, Q}^{M, N}\left[\left.\min \left\{t_{1}, \ldots, t_{n}\right\}\right|_{\left(b_{Q}, \beta_{Q}\right)} ^{\left(a_{P}, \alpha_{P}\right)}\right] d t .
\end{aligned}
$$

Applying now (3.9) of Lemma 3.2, and the following result giving the Mellin transform of the H-function [1, equation (E.20), page 348], namely,

$$
\begin{aligned}
\left\{H_{P, Q}^{M, N}\left[\left.x\right|_{\left(b_{Q}, \beta_{Q}\right)} ^{\left(a_{P}, \alpha_{P}\right)}\right]\right\}^{*}(s)= & \frac{\prod_{j=1}^{M} \Gamma\left(b_{j}+\beta_{j} s\right) \prod_{i=1}^{N} \Gamma\left(1-a_{i}-\alpha_{i} s\right)}{\prod_{i=N+1}^{P} \Gamma\left(a_{i}+\alpha_{i} s\right) \prod_{j=M+1}^{Q} \Gamma\left(1-b_{j}-\beta_{j} s\right)} \\
& \times\left(-\min _{1 \leq j \leq M}\left[\frac{\operatorname{Re}\left(b_{j}\right)}{\beta_{j}}\right]<\operatorname{Re}(s)<\min _{1 \leq i \leq N}\left[\frac{1-\operatorname{Re}\left(a_{i}\right)}{\alpha_{i}}\right]\right),
\end{aligned}
$$

we obtain

$$
\begin{aligned}
& \left(\begin{array}{l|l}
\mathbf{H}_{\mathbf{P}, \mathbf{Q},+; \mathbf{n}}^{\mathrm{M}, \mathbf{N}} & \begin{array}{l}
\left(\mathbf{a}_{\mathrm{p}}, \alpha_{\mathrm{P}}\right) \\
\left(\mathbf{b}_{\mathbf{Q}}, \beta_{\mathbf{Q}}\right)
\end{array} \\
\mathbf{x}^{-\mathbf{s}}
\end{array}\right)(x) \\
& =\frac{\Gamma(1+n-|s|) \prod_{j=1}^{M} \Gamma\left(b_{j}+\beta_{j}(|s|-n)\right) \prod_{i=1}^{N} \Gamma\left(1-a_{i}-\alpha_{i}(|s|-n)\right)}{\Gamma(n-|s|) \prod_{i=N+1}^{P} \Gamma\left(a_{i}+\alpha_{i}(|s|-n)\right) \prod_{j=M+1}^{Q} \Gamma\left(1-b_{j}-\beta_{j}(|s|-n)\right)} x^{-s} .
\end{aligned}
$$

Similarly, by using the multidimensional operator (3.2), we obtain

$$
\begin{aligned}
& \left(\begin{array}{l|l}
\mathbf{H}_{\mathbf{P}, \mathbf{Q},-; \mathbf{n}}^{\mathrm{M}, \mathbf{N}} & \begin{array}{l}
\left(\mathbf{a}_{\mathrm{P}}, \alpha_{\mathrm{P}}\right) \\
\left(\mathbf{b}_{\mathbf{Q}}, \beta_{\mathbf{Q}}\right)
\end{array} \\
\mathbf{x}^{-\mathbf{s}}
\end{array}\right)(x) \\
& =\frac{(-1)^{n} \Gamma(1-n+|s|) \prod_{j=1}^{M} \Gamma\left(b_{j}+\beta_{j}(|s|-n)\right) \prod_{i=1}^{N} \Gamma\left(1-a_{i}-\alpha_{i}(|s|-n)\right)}{\Gamma(-n+|s|) \prod_{i=N+1}^{P} \Gamma\left(a_{i}+\alpha_{i}(|s|-n)\right) \prod_{j=M+1}^{Q} \Gamma\left(1-b_{j}-\beta_{j}(|s|-n)\right)} x^{-s} .
\end{aligned}
$$

The result (3.13) on specializing the parameters in accordance with (3.5) yields the formula [7, equation (3.6), page 148] involving the multidimensional modified integral operator (1.4). Similarly, we can deduce a result from (3.12) which involves the multidimensional modified integral operator (1.3).

\section{Acknowledgments}

The author is thankful to the referees for suggestions. The present investigation was supported by All India Council for Technical Education (AICTE), Government of India, New Delhi. 


\section{References}

[1] V. S. Kiryakova, Generalized Fractional Calculus and Applications, Pitman Research Notes in Mathematics Series, vol. 301, Longman Scientific \& Technical, Harlow, 1994.

[2] A. P. Prudnikov, Yu. A. Brychkov, and O. I. Marichev, Integrals and Series, Vol. 3, More Special Functions, Gordon and Breach, New York, 1990.

[3] R. K. Raina, A note on multidimensional modified fractional calculus operators, Proceedings of the Indian Academy of Sciences. Mathematical Sciences 106 (1996), no. 2, 155-162.

[4] R. K. Raina and P. Kr. Chhajed, A note on multidimensional fractional calculus operators involving Gauss hypergeometric functions, Kyungpook Mathematical Journal 45 (2005), no. 1, 1-11.

[5] S. G. Samko, A. A. Kilbas, and O. I. Marichev, Fractional Integrals and Derivatives: Theory and Applications, Gordon and Breach, Yverdon, 1993.

[6] V. K. Tuan, R. K. Raina, and M. Saigo, Multidimensional fractional calculus operators, International Journal of Mathematical and Statistical Sciences 5 (1996), no. 2, 141-160.

[7] V. K. Tuan and M. Saigo, Multidimensional modified fractional calculus operators involving the Gauss hypergeometric function, Mathematische Nachrichten 161 (1993), 253-270.

[8] __ Modified fractional integral operators in $L_{p}$ space with power-logarithmic weight, Mathematica Japonica 49 (1999), no. 1, 145-150.

R. K. Raina: Department of Mathematics, College of Technology and Engineering, MP University of Agriculture and Technology, Udaipur 313 001, Rajasthan, India

E-mail address: rainark_7@hotmail.com 


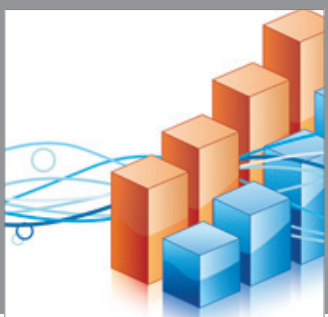

Advances in

Operations Research

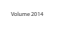

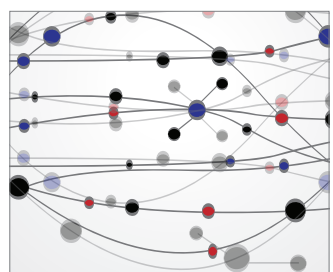

\section{The Scientific} World Journal
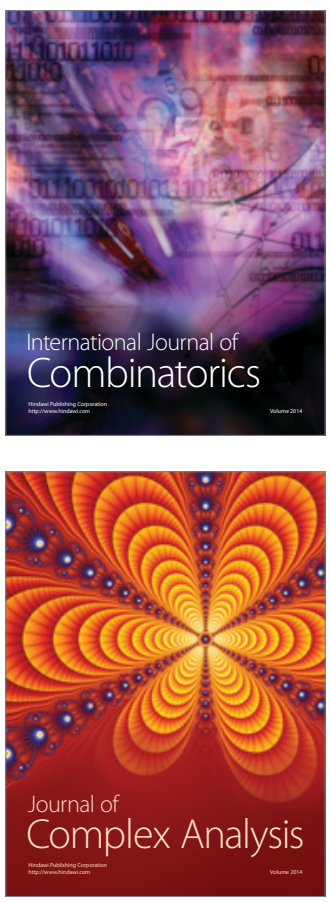

International Journal of

Mathematics and

Mathematical

Sciences
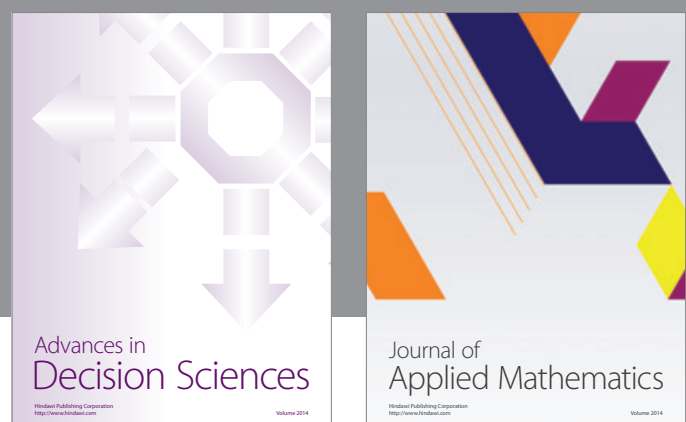

Journal of

Applied Mathematics
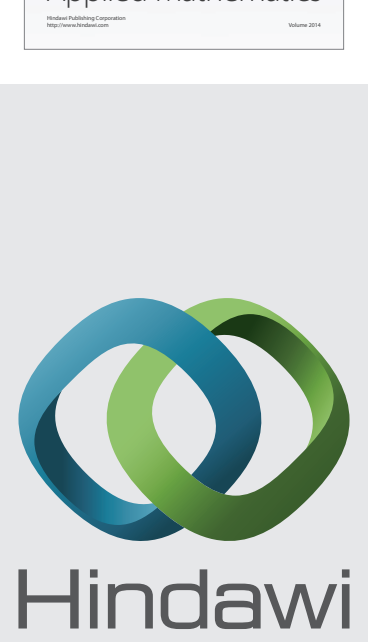

Submit your manuscripts at http://www.hindawi.com
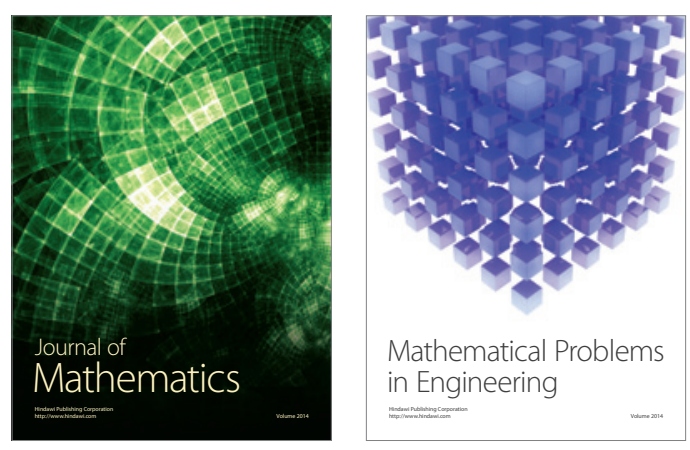

Mathematical Problems in Engineering
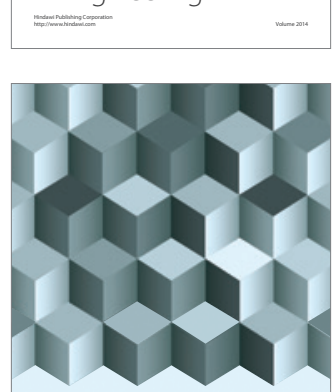

Journal of

Function Spaces
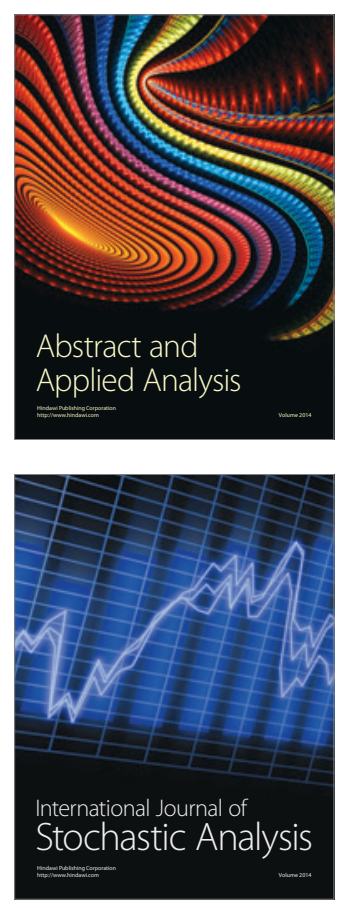

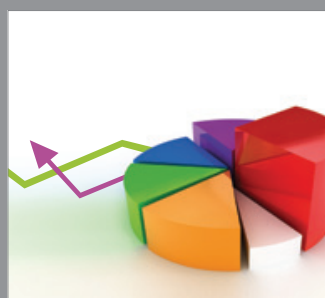

ournal of

Probability and Statistics

Promensencen
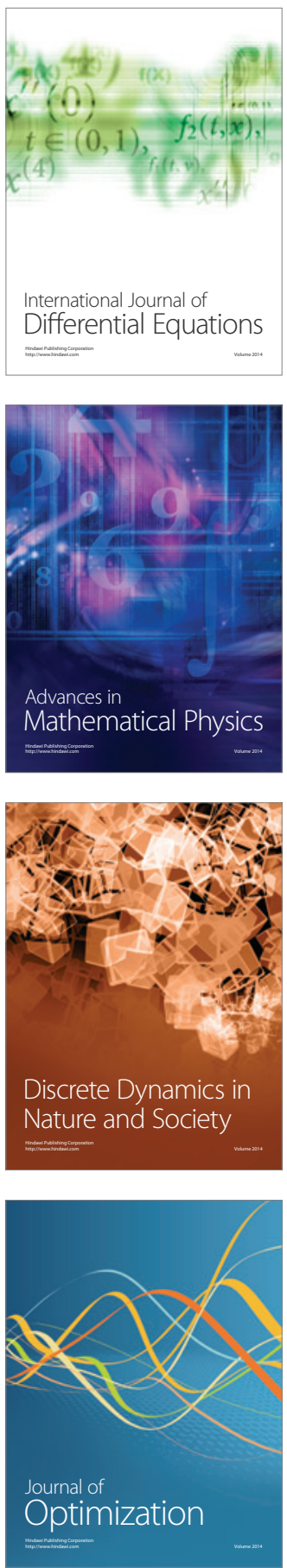